\title{
Order Submission Strategy and the Curious Case of Marketable Limit Orders
}

\author{
Mark Peterson and Erik Sirri*
}

\begin{abstract}
We provide empirical evidence on order submission strategy of investors with similar commitments to trade by comparing the execution costs of market orders and marketable limit orders (i.e., limit orders with the same trading priority as market orders). The results indicate the unconditional trading costs of marketable limit orders are significantly greater than market orders. We attribute the difference in costs to a selection bias and provide evidence suggesting the order submission strategy decision is based on prevailing market conditions and stock characteristics. After correcting for the selection bias, the results show the average trader chooses the order type with lower conditional trading costs.
\end{abstract}

\section{Introduction}

Investors face an order submission strategy decision every time they trade. They may submit market orders or, alternatively, limit orders. Each order type has advantages and disadvantages. A market order demands immediacy, requiring execution as soon as practicable, at the best available price. However, the execution price is uncertain at the time the order is submitted. This is particularly true, for example, when the order is for more than the quoted depth. Therefore, market order traders face some price risk. On the other hand, a limit order allows a trader to set a limit price at which the order might fill, but there is a risk the order does not execute. Understandably, order submission strategy is very important as traders in financial markets encounter the choice of order type hundreds of thousands of times per day.

*Peterson, map1@cba.siu.edu, Department of Finance, Southern Illinois University, Carbondale, IL 62901; Sirri, sirri@babson.edu, Finance Department, Babson College, Wellesley, MA 02157. We appreciate comments from Gordon Alexander, Scott Gilbert, Jeff Harris, Marc Lipson, Evren Örs, Venkatesh Panchapagesan, Robert Van Ness, and Larry Harris (the referee). Comments by seminar participants at the 2000 FMA Meetings, 2000 Nasdaq-Notre Dame Microstructure Conference, 2001 Western Finance Association Meetings, 2000 NBER Market Microstructure Meeting, and Southern Illinois University are gratefully acknowledged. Parts of this work were completed when the authors were with the Securities and Exchange Commission. The SEC, as a matter of policy, disclaims any responsibility for any private publication or statements by any of its employees. The views expressed herein are those of the authors' and do not necessarily reflect the views of the Commission, or of the authors' former colleagues on the staff of the Commission. Any errors that may remain are our own. Peterson acknowledges financial support from the SIU College of Business Summer Scholars Program. 
Microstructure research in modeling the trader's choice between market orders and limit orders has focused on the tradeoff between execution certainty and transactions costs (see, e.g., Cohen, et al. (1981), Harris (1997), Harris and Hasbrouck (1996), Holden and Chakravarty (1995), and Kumar and Seppi (1992)). Among the important empirical results found in Harris and Hasbrouck (1996) are that limit orders perform better (based on their ex ante performance measure) and the limit order placement strategies most commonly used perform best. The results in Harris and Hasbrouck (1996) are conditioned on order size, bid-ask spread, and order direction, and assume traders using market orders and limit orders have the same level of commitment to trade.

In this paper, we extend the work of Harris and Hasbrouck (1996) by investigating order submission strategy of traders on the New York Stock Exchange (NYSE) with similar commitments to trade. In so doing, we compare the trading costs of market orders and marketable limit orders. A marketable limit order is a priced order with the limit price set at, or better than, the opposite side quote (bid price for sell orders and ask price for buy orders). Marketable limit orders have the same trading priority as market orders because the NYSE prioritizes orders on price. The only difference between a market order and a marketable limit order is the limit order has an execution price bounded by the limit price. Thus, this analysis provides insight into order submission strategy when price priority is not an issue. In this respect, our research allows us to investigate the benefits of one of the characteristics of limit orders, namely, a bounded execution price.

Interestingly, previous studies including Angel (1997), Harris and Hasbrouck (1996), and SEC (1997) have identified significant differences in execution quality between market and marketable limit orders. These studies indicate traders using the marketable limit order strategy pay greater trading costs. The results from these studies are persistent over time, as the trading costs have been calculated using data from 1990 and 1996. Moreover, marketable limit orders appear to perform worse than market orders not only on the NYSE, but on the regional exchanges as well (SEC (1997)). These results are unexpected as there are no institutional reasons why the two order types should be treated differently. If differences in execution quality persist, the survivorship principle dictates investors must receive some other benefit from using marketable limit orders to offset increased execution costs.

The data used in this study include order information (i.e., order type, buy/sell indicator) from the NYSE. ${ }^{1}$ Order data allow an accurate calculation of the relative effective bid-ask spread. Similar to Harris and Hasbrouck (1996), who use data from 1990, we find market orders have lower unconditional trading costs than marketable limit orders.

In an attempt to hold everything else equal, we condition our results on several dimensions, including current market conditions, stock characteristics, and investor type (i.e., individual or non-individual). Further, the choice of order type is not considered to be a random decision and, therefore, a potential selectivity bias exists. The Heckman (1979) two-step estimation technique is employed to

\footnotetext{
${ }^{1}$ Data from the regional exchanges during an earlier time period were also analyzed. These results, available upon request, are qualitatively similar to those reported here. The NYSE-only sample is used because the data are more current and more comprehensive than the data from the regional exchanges.
} 
deal with the selection bias. The results allow the estimation of the value, in terms of differential trading costs, of submitting a marketable limit order instead of a market order, under specified conditions. Other current research uses similar methodology. Madhavan and Cheng (1997) compare trades in the upstairs and downstairs markets, and Handa, Schwartz, and Tiwari (1999) compare trades from the floor to system entered trades.

Our findings are consistent with investors selecting the order type based on current stock and market conditions, choosing the strategy minimizing trading costs. In particular, we show investors strategically place marketable limit orders to reduce price risk in cases when trading imbalances exist and the potential for receiving a fill at an unfavorable price increases. We find marketable limit orders are used proportionally more often: i) for larger orders, ii) by non-individual investors, iii) when the order size exceeds the quoted depth, iv) when quote imbalances exist, v) when the depth is relatively low, and vi) when spreads are narrow. Overall, the evidence indicates investors optimize their trading strategy where the type of order is an important consideration. We also show that, although the average investor chooses an optimal trading strategy, there are cases when investors choose sub-optimal strategies. In general, we find evidence consistent with individual investors being less likely to choose optimal order submission strategies.

\section{Data Description}

Order data were obtained from the NYSE's System Order Database (SOD) for two weeks from June 30, 1997, to July 11, 1997. Hasbrouck (1992) describes the SOD file in detail. During this time period the minimum price variation, or tick, was $\$ 1 / 16$ for most stocks. The analysis is conducted only for NYSE-listed issues of ordinary common shares as identified in the CRSP database. Orders entered outside of normal trading hours (9:30AM to 4:00PM EST) are excluded. This requirement is made because our cost estimator requires a benchmark quote. Opening orders, tick sensitive orders, market orders with price qualifiers, and limit orders that are not marketable are also excluded. As a result of these screens, we consider two types of orders: regular way market orders and marketable limit orders. Of the marketable limit orders, $91 \%, 6 \%$, and $2 \%$ have a limit price equal to, $\$ 1 / 16$ better than, and $\$ 1 / 8$ better than the opposite side quote, respectively. We examined whether orders with the placement of the limit price beyond the market quote were any different from the rest of the limit orders. The results did not indicate these orders performed any differently.

The SOD file is valuable because it includes comprehensive data on orders submitted through the NYSE's SuperDot system. Among other items, the SOD file includes information on the account type originating the order. This information is useful as one could determine whether the order originated from a member (as principal or agent), an individual investor, or any other customer including institutions, non-member broker/dealers, and managed accounts. Despite its comprehensive description of orders, the SOD file does not represent the entire picture of trading on the NYSE. Sofianos and Werner (2000) examine trading activity of NYSE floor brokers and find floor broker participation is as high as $44 \%$. They conclude it is misleading to make inferences concerning liquidity using only SOD 
and TAQ data. Ross, Shapiro, and Smith (1996) have reported over $80 \%$ of the NYSE orders, accounting for $30-40 \%$ of the volume, are executed through SuperDOT. Hence, an important caveat should be considered at this point: order submission strategies described here apply to system orders sent to an auction market, not necessarily to orders or trades made by floor brokers, or orders sent to a dealer market such as Nasdaq.

Benchmark quotes are required to accurately estimate transactions costs. Quotes are available from the TAQ database. The national best bid and offer (NBBO) were calculated at each point in time for all of the sample stocks over the sample period. If a stock traded in multiple markets and more than one market was at the best quote, the market with the highest depth was considered to have the best quote. The order data were then merged with quote data to create an observation with all the required information. The order data include information such as the order arrival time, the size of the order, a buy/sell indicator, an indicator to identify market and limit orders, and limit order prices. Information on the execution of the order includes the time of the execution, the execution price, and the fill quantity. For orders filled in more than one piece, the data contain information for each of the fills and a volume-weighted execution price is calculated.

Many factors may jointly determine order submission strategy, including the size of the order and the level of investor sophistication. As the size of the order increases, the importance of the order type decision increases because any errors in order type selection will increase with order size. More sophisticated investors may more carefully consider the order type decision if they are able to recognize the marginal benefits from using an optimal order submission strategy. Therefore, throughout our analysis we categorize our results on these two potential factors. Specifically, we categorize our results based on the relative order size, i.e., whether the order size is greater than the depth and by the account type as reported in the SOD ACCTYP field. The ACCTYP variable identifies whether the order originated from an individual investor's account as opposed to an order coming from an institution, managed account, or other proprietary account. We assume the level of investor sophistication may vary between individual investors and the complement set of investors (referred to here as non-individual investors).

Table 1, panel A reports the number of orders. In all, we analyze 1,493,093 orders of which $932,000(62 \%)$ are market orders and 561,093 (38\%) are marketable limit orders. In general, the usage of limit orders increases (on a percentage basis) with increases in order size. Small orders (100-500 shares), medium orders (501-5,000 shares), and large orders ( $>5,000$ shares) are marketable limit orders for $23 \%, 54 \%$, and $73 \%$ of the observations, respectively. This result indicates order size is a determinant of order type selection. Further, marketable limit orders are used more frequently when the size of the order exceeds the current quoted depth as $34 \%$ of the orders with order size less than or equal to the opposite side posted depth are marketable limit orders, but $58 \%$ of the orders with order size greater than the opposite side posted depth are marketable limit orders. However, this may be due to order size effects, rather than market depth effects.

Many of the interesting patterns in Table 1, panel A also appear in panels $\mathrm{B}$ and $\mathrm{C}$, which categorize orders as coming from individual investors or nonindividual investors. We observe that individual investors tend to use market or- 
TABLE 1

Number of Orders

\begin{tabular}{|c|c|c|c|c|c|c|}
\hline \multirow[b]{2}{*}{ Order Size } & \multicolumn{2}{|c|}{ All } & \multicolumn{2}{|c|}{$\begin{array}{l}\text { Order Size } \\
\leq \text { Depth }\end{array}$} & \multicolumn{2}{|c|}{$\begin{array}{c}\text { Order Size } \\
>\text { Depth }\end{array}$} \\
\hline & Number & $\%$ Limit & Number & $\%$ Limit & Number & \% Limit \\
\hline \multicolumn{7}{|c|}{ Panel A. All Orders } \\
\hline $\begin{array}{l}\text { Small } \\
\text { Medium } \\
\text { Large } \\
\text { All }\end{array}$ & $\begin{array}{r}819,827 \\
620,602 \\
52,664 \\
1,493,093\end{array}$ & $\begin{array}{l}23 \% \\
54 \% \\
73 \% \\
38 \%\end{array}$ & $\begin{array}{r}792,555 \\
456,627 \\
21,002 \\
1,270,184\end{array}$ & $\begin{array}{l}23 \% \\
52 \% \\
74 \% \\
34 \%\end{array}$ & $\begin{array}{r}27,272 \\
163,975 \\
31,662 \\
222,909\end{array}$ & $\begin{array}{l}38 \% \\
58 \% \\
73 \% \\
58 \%\end{array}$ \\
\hline \multicolumn{7}{|c|}{ Panel B. Orders from Non-Individual Investors } \\
\hline $\begin{array}{l}\text { Small } \\
\text { Medium } \\
\text { Large } \\
\text { All }\end{array}$ & $\begin{array}{r}508,661 \\
525,318 \\
48,828 \\
1,082,807\end{array}$ & $\begin{array}{l}32 \% \\
59 \% \\
74 \% \\
47 \%\end{array}$ & $\begin{array}{r}487,329 \\
381,866 \\
19,413 \\
888,608\end{array}$ & $\begin{array}{l}31 \% \\
58 \% \\
75 \% \\
44 \%\end{array}$ & $\begin{array}{r}21,332 \\
143,452 \\
29,415 \\
194,199\end{array}$ & $\begin{array}{l}45 \% \\
61 \% \\
74 \% \\
61 \%\end{array}$ \\
\hline \multicolumn{7}{|c|}{ Panel C. Orders from Individual Investors } \\
\hline $\begin{array}{l}\text { Small } \\
\text { Medium } \\
\text { Large } \\
\text { All }\end{array}$ & $\begin{array}{r}311,166 \\
95,284 \\
3,836 \\
410,286\end{array}$ & $\begin{array}{r}8 \% \\
25 \% \\
61 \% \\
13 \%\end{array}$ & $\begin{array}{r}305,226 \\
74,761 \\
1,589 \\
381,576\end{array}$ & $\begin{array}{c}8 \% \\
22 \% \\
56 \% \\
11 \%\end{array}$ & $\begin{array}{r}5,940 \\
20,523 \\
2,247 \\
28,710\end{array}$ & $\begin{array}{l}13 \% \\
33 \% \\
64 \% \\
31 \%\end{array}$ \\
\hline
\end{tabular}

Data are taken from the NYSE's System Order Database file over 6/30/97-7/11/97 and include orders from stocks identified in the CRSP names structure as ordinary common shares. Orders entered when the NBBO was unavailable, or when the NBBO was less than $\$ 1 / 16$ or greater than $\$ 1 / 4$ were excluded. Small orders are for 100-500 shares. Medium orders are for 501-5,000 shares. Large orders are for more than 5,000 shares. Order Size $\leq$ Depth refers to orders with size less than or equal to the opposite side posted depth. Order Size $>$ Depth refers to orders with size greater than the opposite side posted depth.

ders proportionally more than non-individuals. This result may be partially explained by the fact that orders from individual investors tend to be smaller. This result may also suggest individual investors pursue different order submission strategies than non-individual investors or that individuals trade different stocks. The data in Table 1, taken together with models such as Easley and O'Hara (1987) where order size affects trading costs, are consistent with the marginal marketable limit order trader being more sophisticated than the marginal market order trader. If this conjecture is true, we should notice more evidence of order submission strategies for larger orders from non-individual investors.

\section{Empirical Analysis of Trading Costs, Market Liquidity, and Volatility}

\section{A. Measurement of Trading Costs by Order Type}

The measure of execution costs we use is the relative effective spread defined as

$$
\text { Relative effective spread }=2 \times D \times(P-\mathrm{MP}) / \mathrm{MP} \text {, }
$$

where $D$ is the trade direction: 1 for buys, -1 for sells; $P$ is the execution price (for orders with multiple fills we use the volume-weighted trade price); and MP is the midpoint of the quotes at the order arrival time. The relative effective spread represents the trading costs, on a percentage basis, less commissions. Order data 
are critical to correctly identify the trade direction as the practice of price improvement occurs frequently in order-driven markets such as the NYSE (Ross, Shapiro, and Smith (1996)). The use of these data is especially important because more than $53 \%$ of the orders are submitted in minimum variation markets, situations in which one cannot detect price improvement, hence trade direction.

In some cases, orders were not completely filled. Many of these orders were marketable limit orders cancelled shortly (within one minute) after order submission. In such cases, we assigned a price equal to the weighted average of the current quote and the price one tick outside the quote, with the weight on the latter being equal to the percent by which the order size exceeds the quoted size. Bacidore, Battalio, and Jennings ((2000), p. 14) note "the liquidity supply function may be so steep or the order size so great that the order would execute at multiple prices outside the quote without floor intervention." If this is the case, then our execution cost estimator would underestimate the imputed cost of the order. On the other hand, the estimated execution, as described above, would not necessarily allow the order the opportunity of price improvement and, therefore, may produce an imputed cost that would be overestimated. In any event, the occurrence of an unexecuted market or marketable limit order is rare, so any problem with imputing costs should be minimal. Indeed, similar results, available upon request, were found when unexecuted orders were excluded.

Table 2 reports the average relative effective spreads. ${ }^{2}$ Panel A includes the results from all orders, regardless of investor type. Overall, the average relative effective spread for market orders is $0.240 \%$; for marketable limit orders it is $0.260 \%$. The difference of $0.020 \%$ is statistically and economically significant, and is consistent with the studies previously cited. There appears to be a size effect as small (large) market orders are less (more) costly than small (large) marketable limit orders. Not surprisingly, Table 2 also shows market orders have lower average relative effective spreads when the order size is less than or equal to the opposite side posted depth, although the difference in costs is economically small. When the order size is more than the opposite side posted depth, marketable limit orders have lower average relative effective spreads. Panel B of Table 2 includes orders from non-individual investors accounts. For these orders, the patterns are very similar to the results summarized in panel $\mathrm{A}$.

Turning to Table 2, panel $\mathrm{C}$, orders from individual investor accounts, we notice that, in general, the trading costs of the limit order strategy are significantly higher than the market order strategy. Interestingly, the differences are quite large economically, even for small orders for less than the quoted depth $(\sim 0.1 \%)$. We have no immediate explanation for this result as there may be characteristics other than order size and quoted depth that determine trading costs. Comparing the results in panel $\mathrm{B}$ to the results in panel $\mathrm{C}$, the data seem to indicate individual investors pay higher trading costs than non-individual investors. However, under

\footnotetext{
${ }^{2}$ Buy orders and sell orders were analyzed separately. However, because the relative effective spreads of buy orders were very similar to the relative effective spreads of sell orders, results are not reported separately to conserve space.
} 
TABLE 2

Average Relative Effective Spread (\%)

\begin{tabular}{|c|c|c|c|c|c|c|}
\hline \multirow[b]{2}{*}{$\underline{\text { Order Size }}$} & \multicolumn{2}{|c|}{ All } & \multicolumn{2}{|c|}{$\begin{array}{c}\text { Order Size } \\
\leq \text { Depth }\end{array}$} & \multicolumn{2}{|c|}{$\begin{array}{c}\text { Order Size } \\
>\text { Depth }\end{array}$} \\
\hline & Market & Limit & Market & Limit & Market & Limit \\
\hline \multicolumn{7}{|c|}{ Panel A. All Orders } \\
\hline Small & $0.199 \%$ & $0.205 \% * *$ & $0.195 \%$ & $0.201 \%$ ** & $0.315 \%$ & $0.261 \%$ ** \\
\hline Medium & 0.321 & $0.269^{* *}$ & 0.280 & $0.231^{\star *}$ & 0.449 & $0.363^{\star *}$ \\
\hline Large & 0.490 & $0.457^{* *}$ & 0.448 & $0.343^{\star *}$ & 0.516 & 0.534 \\
\hline All & 0.240 & $0.260^{\star *}$ & 0.219 & $0.223^{\star *}$ & 0.431 & $0.386^{* *}$ \\
\hline \multicolumn{7}{|c|}{ Panel B. Orders from Non-Individual Investors } \\
\hline Small & $0.182 \%$ & $0.187 \%$ ** & $0.178 \%$ & $0.183 \%$ ** & $0.282 \%$ & $0.248 \%$ ** \\
\hline Medium & 0.271 & $0.242^{* \star}$ & 0.227 & $0.203^{\star *}$ & 0.397 & $0.341^{* \star}$ \\
\hline Large & 0.433 & 0.418 & 0.350 & $0.298^{\star *}$ & 0.484 & 0.499 \\
\hline All & 0.220 & $0.237^{* *}$ & 0.195 & $0.199^{* *}$ & 0.388 & $0.362^{\star *}$ \\
\hline \multicolumn{7}{|c|}{ Panel C. Orders from Individual Investors } \\
\hline Small & $0.219 \%$ & $0.317 \%$ ** & $0.216 \%$ & $0.313 \%$ ** & $0.389 \%$ & $0.431 \%$ * \\
\hline Medium & 0.471 & $0.617^{\star *}$ & 0.426 & $0.601^{* *}$ & 0.659 & 0.656 \\
\hline Large & 0.963 & 1.066 & 1.128 & 1.078 & 0.823 & $1.059^{\star *}$ \\
\hline All & 0.273 & $0.487^{* *}$ & 0.254 & $0.442^{* *}$ & 0.595 & $0.701^{* *}$ \\
\hline
\end{tabular}

Data are taken from the NYSE's System Order Database file over 6/30/97-7/11/97 and include orders from stocks identified in the CRSP names structure as ordinary common shares. The relative effective spread is calculated as $2 \times D \times$ (Price - Quote Midpoint)/Quote Midpoint, where $D$ is 1 for buys and -1 for sells. The NBBO is measured at order arrival time. Orders entered when the NBBO was unavailable (e.g., the open) or was less than $\$ 1 / 16$ or greater than $\$ 1 / 4$ were excluded. Orders that are not completely filled have an imputed price of the opposite side quote for shares up to the current depth and $\$ 1 / 16$ more(less) for the remaining shares for buys(sells). Small orders are for 100-500 shares. Medium orders are for $501-5,000$ shares. Large orders are for more than 5,000 shares. Order Size $\leq$ Depth refers to orders with size less than or equal to the opposite side posted depth. Order Size $>$ Depth refers to orders with size greater than the opposite side posted depth. ${ }^{*}$ and ${ }^{* *}$ indicate the difference in relative effective spreads across order types is significant at the $5 \%$ and $1 \%$ level, respectively, using a $t$-test.

closer inspection, we found individuals tend to trade lower priced, less liquid stocks than non-individuals. ${ }^{3}$

To summarize the results in Table 2, we find the average marketable limit order trader incurs larger transactions costs. The difference in costs is related to order size, investor type, and the size of the order relative to the opposite side quoted depth. Additionally, although the average marketable limit order appears to be more expensive, these orders tend to be used more often (recall Table 1) when the average marketable limit order is less expensive than the average market order.

\section{B. Depth at the Quotes}

Biais, Hillion, and Spatt (1995) examine order flow on the Paris Bourse and provide results consistent with the presence of limit order traders monitoring the order book, competing to provide liquidity to the market when it is needed and rewarded, and quickly seizing favorable trading opportunities. For the orders in this study, which are orders entered through a computer system and not from the

\footnotetext{
${ }^{3}$ In a previous draft, we tested whether individuals paid higher transactions costs, all else equal. We found the average marketable limit order submitted by individuals incurred additional costs relative to orders from non-individuals of approximately $3 d$ per round-trip, but market orders submitted by individuals incurred smaller costs relative to orders from non-individuals, on the order of approximately $2 d$ per round-trip.
} 
floor, it is likely that quotations from the Consolidated Quotation System (CQS) are the primary source of current stock liquidity. The CQS quotes not only include prices, but also the number of shares honored at those prices. Investors who may otherwise choose to use a market order may choose to use a limit order if the quoted depth is relatively small.

Table 3, panel A, reports the percentage of orders for which the number of shares is greater than the posted depth at the opposite side quote. For both individuals and non-individuals, the percentage of marketable limit orders with the number of shares exceeding the quoted depth is significantly greater than the percentage of market orders with the number of shares exceeding the quoted depth. This result is consistent with traders monitoring the quotes and using a marketable limit order strategy when there is a significant risk a part of the order might execute at the next price step. This result is interesting because it is consistent with investors, including individual investors, using the quotes as an input into their order submission decision framework.

Table 3, panel B includes another measure of depth, which we refer to as quote imbalance. The quote imbalance is defined as

$$
\text { Imbalance }=2 \times(\text { Bid size }- \text { Ask size }) /(\text { Bid size }+ \text { Ask size }) \text {. }
$$

TABLE 3

Analysis of Depth at the Opposite Quote at Order Submission Time

\begin{tabular}{|c|c|c|c|c|}
\hline & \multicolumn{2}{|c|}{$\begin{array}{c}\text { Non-Individual } \\
\text { Investors }\end{array}$} & \multicolumn{2}{|c|}{$\begin{array}{l}\text { Individual } \\
\text { Investors }\end{array}$} \\
\hline & Market & Limit & Market & Limit \\
\hline \multicolumn{5}{|c|}{ Panel A. \% Greater than Depth } \\
\hline All orders & $13 \%$ & $23 \%$ ** & $5 \%$ & $17 \% \%^{* *}$ \\
\hline \multicolumn{5}{|c|}{ Panel B. Median Imbalance } \\
\hline $\begin{array}{l}\text { Buy orders } \\
\text { Small } \\
\text { Medium } \\
\text { Large } \\
\text { All }\end{array}$ & $\begin{array}{r}0.00 \\
0.00 \\
-0.17 \\
0.00\end{array}$ & $\begin{array}{l}0.71^{\star *} \\
0.52^{\star \star} \\
0.00^{\star *} \\
0.60^{\star *}\end{array}$ & $\begin{array}{r}-0.13 \\
0.00 \\
0.00 \\
-0.11\end{array}$ & 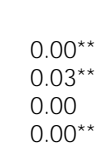 \\
\hline $\begin{array}{l}\text { Sell orders } \\
\text { Small } \\
\text { Medium } \\
\text { Large } \\
\text { All }\end{array}$ & $\begin{array}{r}-0.14 \\
-0.20 \\
0.00 \\
-0.17\end{array}$ & $\begin{array}{l}-1.00^{\star \star} \\
-0.73^{\star \star} \\
-0.25^{\star \star} \\
-0.78^{\star \star}\end{array}$ & $\begin{array}{l}0.00 \\
0.00 \\
0.00 \\
0.00\end{array}$ & $\begin{array}{l}-0.06^{\star *} \\
-0.21^{\star *} \\
-0.16^{\star *} \\
-0.15^{\star *}\end{array}$ \\
\hline \multicolumn{5}{|c|}{ Panel C. Order Type Usage vs. Relative Depth } \\
\hline $\begin{array}{l}\text { Small depth } \\
\text { Medium depth } \\
\text { Large depth }\end{array}$ & $\begin{array}{l}47 \% \\
55 \% \\
61 \%\end{array}$ & $\begin{array}{l}53 \% \\
45 \% \\
39 \%\end{array}$ & $\begin{array}{l}81 \% \\
82 \% \\
83 \%\end{array}$ & $\begin{array}{l}19 \% \\
18 \% \\
17 \%\end{array}$ \\
\hline \multicolumn{5}{|c|}{$\begin{array}{l}\text { Data are taken from the NYSE's System Order Database file over } 6 / 30 / 97-7 / 11 / 97 \text {, and include orders } \\
\text { from stocks identified in the CRSP names structure as ordinary common shares. \% Greater than Depth } \\
\text { refers to orders submitted for more shares than are at the NBBO depth. Imbalance is calculated as } \\
2 \times \text { (Bid depth - Ask depth)/(Bid depth + Ask depth). Small orders are for } 100-500 \text { shares. Medium } \\
\text { orders are for } 501-5,000 \text { shares. Large orders are for more than } 5,000 \text { shares. Order Type Usage vs. } \\
\text { Relative Depth is the median percentage order type usage, across stocks, controlling for each stock's } \\
\text { depth. Small depth indicates the depth for the lowest third of observations. Medium depth indicates } \\
\text { the depth for the middle third of observations. Large depth indicates the depth for the highest third of } \\
\text { observations. }{ }^{*} \text { and }{ }^{* *} \text { indicate the percentage of orders greater than the depth (imbalance) across order } \\
\text { types is significant at the } 5 \% \text { and } 1 \% \text { levels, respectively, using a binomial } z \text {-test (Wilcoxon signed rank } \\
\text { test). }\end{array}$} \\
\hline
\end{tabular}


The data indicate marketable limit orders are used proportionally more often in the presence of a quote imbalance and market orders are used when the quoted depths are relatively even. For example, buy orders from non-individual investors have a median imbalance of 0.60 for marketable limit orders, but a median imbalance of 0.00 for market orders. These imbalances are significantly different at the $1 \%$ level using a Wilcoxon signed rank test. The corresponding median imbalance for marketable limit sell orders from non-individuals is -0.78 , but -0.17 for market sell orders. One explanation for traders using marketable limit orders when imbalances exist is traders may believe the quotes are about to move. For buy orders, investors viewing the quoted depths and realizing the bid size is significantly greater than the ask size, might assume there are more buyers than sellers. Hence, they may believe the quotes move up. Traders, who otherwise might choose to use a market order, may choose a limit order so they eliminate the likelihood of executing at the next price step.

Interestingly, it appears imbalance matters most for smaller orders. However, if one recognizes the frequency of limit order usage increases with order size, this result is not entirely unexpected. In other words, order size may dominate imbalance in determining order submission strategy. Overall, the data indicate as the bid depth exceeds the ask depth, limit orders are used more frequently for buy orders. The converse is true for sell orders. The same patterns exist for orders from individual investors. However, the level of quote imbalance is considerably smaller.

In Table 3, panel $\mathrm{C}$, we control for stock effects by examining order type selection conditioning on each stock's relative depth. For each stock we determine cutoff points for small, medium, and large depth by examining the distribution of trade-weighted quoted depths. Then, we calculate for each stock and level of depth, the percentage of limit order usage. The cross-sectional median percentage limit order usage is reported in the table. In general, limit order usage increases with a decrease in the relative depth. For non-individual investors, we note 53\% of the orders submitted when the depth was small were marketable limit orders. But when the depth is relatively large, only $39 \%$ of the orders were marketable limit orders. As noted above, individual investors use marketable limit orders less frequently. Nevertheless, the pattern of increased limit order usage with a decrease in depth is similar to the pattern from non-individual investors. These data are consistent with traders monitoring the quotes and using this information in considering which order type to use.

Figure 1, A and B, graphically summarizes the results in Table 3 by displaying the frequency distribution of market and marketable limit order usage as a function of order size divided by the opposite side quoted depth. In Figure 1A, the distribution of order size divided by the depth is charted for orders from nonindividual investors. More than $50 \%$ of market orders are for $20 \%$ or less of the posted depth. However, less than $25 \%$ of marketable limit orders are for $20 \%$ or less of the posted depth. Most interesting is the spike at one (order size equals the opposite side quoted depth). Approximately $24 \%$ of the marketable limit orders are for the posted depth and slightly more than $5 \%$ of the market orders are for the posted depth. These data, as above, are consistent with traders monitoring the posted depth. 


\section{FIGURE 1}

1A. Distribution of Order Size/Depth for Orders from Non-Individual Investors Accounts

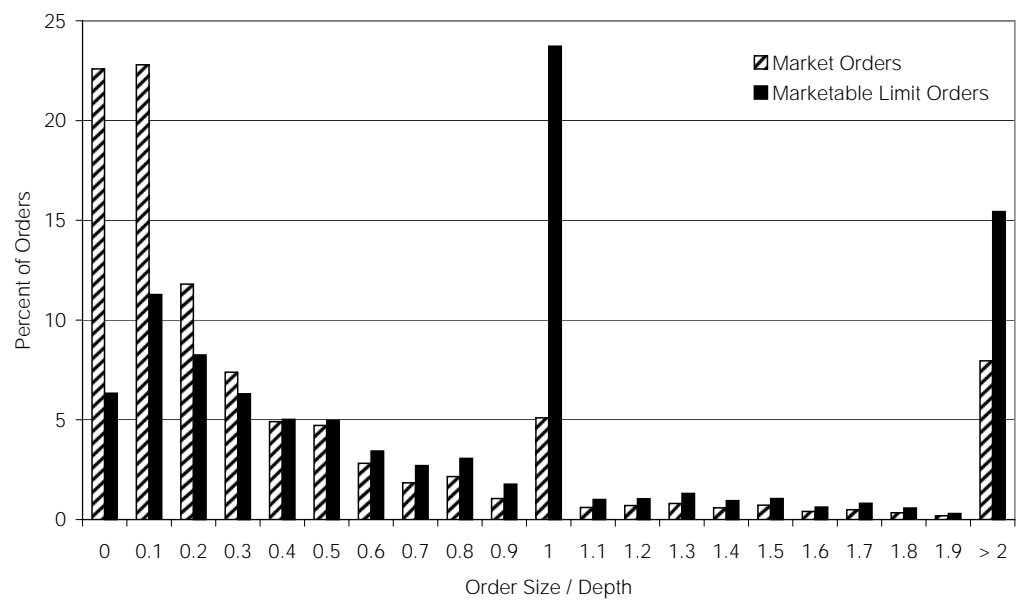

Figure 1A displays the distribution of order size divided by the opposite side quoted depth for orders submitted from accounts identified as other than individuals for the sample of market and marketable limit orders described in Table 1.

1B. Distribution of Order Size/Depth for Orders from Individual Investors Accounts

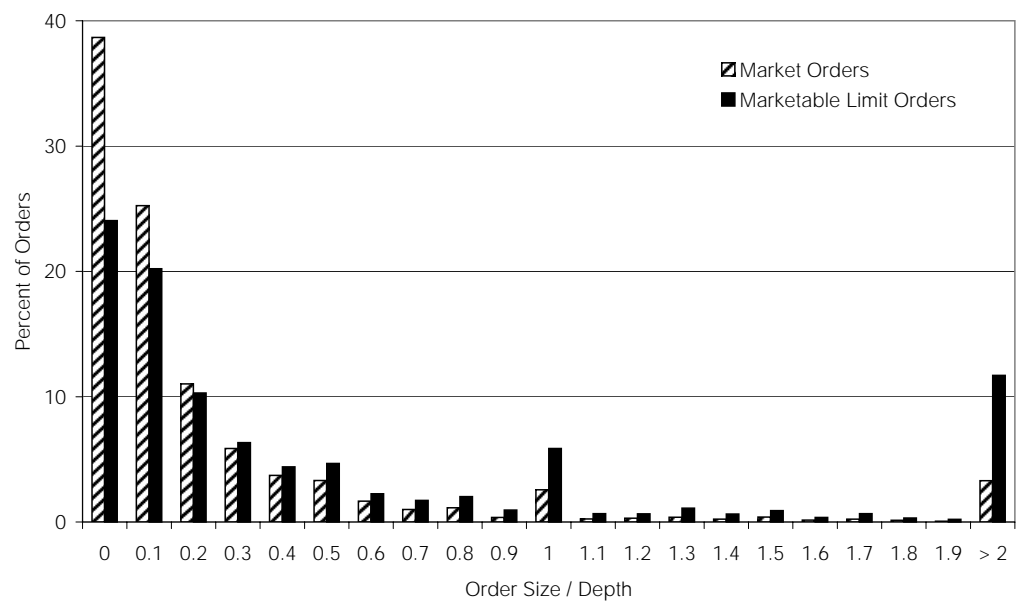

Figure 1B displays the distribution of order size divided by the opposite side quoted depth for orders submitted from accounts identified as from individuals for the sample of market and marketable limit orders described in Table 1. 
Figure 1B plots the distribution of order size divided by the opposite side depth for orders from individual investors. Consistent with the results in Table 3 and Figure 1A, the data indicate, although limit orders are used relatively infrequently, they are used more often as the order size increases relative to the opposite side quote. There is also a spike, albeit not that large, at one, which is consistent with individual investors monitoring the quoted depth.

\section{Stock Volatility}

Harris (1997) derives optimal dynamic order submission strategies for trading problems faced by three stylized traders. He finds traders are most aggressive when volatility is high and when their information advantages are large and decay quickly. Traders are patient when their deadlines are not pressing and when spreads are wide. Traders issue limit orders only if they can capture a portion of the spread. Harris' arguments suggest marketable limit orders will be used more often when spreads are narrow so as to gain price priority. Additionally, limit orders are predicted to be used more often in volatile markets.

Because market order traders are subject to greater price risk than limit order traders, it may be that on more volatile trading days investors would tend to use marketable limit orders to mitigate this risk. We use two proxies for volatility. One proxy, hereafter referred to as short-run volatility, is defined as the squared $\log$ return from the opening trade to the quote midpoint at order submission time, multiplied by 10,000 . The second proxy, hereafter referred to as long-run volatility, is defined as the standard deviation of returns for the previous year for each stock.

Table 4 presents the average volatility for market and marketable limit orders. Panel A reports the results for orders from non-individual investors. Regardless of order size and whether the order size exceeds the opposite side quoted depth, the average short-run volatility is typically higher for market orders than marketable limit orders. The average long-run volatility measure is higher for market orders when the order size is less than the depth, and approximately the same when the order size is greater than the depth. One interpretation of these results is that investors may choose the market order strategy more frequently in volatile markets because the likelihood of non-execution of marketable limit orders increases in such markets. Panel B reports the volatility results for orders from individual investors. For both volatility measures the average volatility is higher for marketable limit orders than market orders.

Because the volatility measures in both panels are significantly different across order types, these data are consistent with traders considering the volatility of the underlying stock when making their order type decision. Another interesting observation is the average short-run volatility is higher for orders exceeding the opposite side quoted depth. This result is consistent with less depth on more volatile trading days. 
TABLE 4

Stock Volatility and Order Submission

\begin{tabular}{|c|c|c|c|c|}
\hline & \multicolumn{2}{|c|}{$\begin{array}{c}\text { Order Size } \\
\leq \text { Depth }\end{array}$} & \multicolumn{2}{|c|}{$\begin{array}{c}\text { Order Size } \\
>\text { Depth }\end{array}$} \\
\hline & Market & Limit & Market & Limit \\
\hline \multicolumn{5}{|c|}{ Panel A. Orders from Non-Individual Investors } \\
\hline \multicolumn{5}{|c|}{ Short-Run Volatility } \\
\hline Small orders & 2.65 & $2.36^{\star *}$ & 3.51 & $2.80^{* *}$ \\
\hline Medium orders & 2.99 & $2.49^{\star \star}$ & 3.17 & $3.01^{* *}$ \\
\hline Large orders & 3.76 & 3.44 & 3.39 & 3.47 \\
\hline All & 2.77 & $2.47^{\star *}$ & 3.25 & $3.08^{* *}$ \\
\hline \multicolumn{5}{|c|}{ Long-Run Volatility $(\times 100)$} \\
\hline Small orders & 1.92 & $1.83^{* *}$ & 1.90 & $1.84^{* *}$ \\
\hline Medium orders & 2.03 & $1.90^{* *}$ & 1.97 & 1.97 \\
\hline Large orders & 2.16 & $2.03^{\star *}$ & 2.07 & $2.09^{* *}$ \\
\hline & 1.96 & $1.88^{\star *}$ & 1.97 & $1.98^{* *}$ \\
\hline \multicolumn{5}{|c|}{ Panel B. Orders from Individual Investors } \\
\hline \multicolumn{5}{|c|}{ Short-Run Volatility } \\
\hline Small orders & 2.74 & $4.24^{* *}$ & 3.35 & $4.41^{*}$ \\
\hline Medium orders & 4.14 & $5.83^{* *}$ & 4.20 & 8.09 \\
\hline Large orders & 9.11 & 7.29 & 4.77 & 19.32 \\
\hline All & 2.99 & $4.93^{\star \star}$ & 4.00 & $9.56^{\star *}$ \\
\hline \multicolumn{5}{|c|}{ Long-Run Volatility $(\times 100)$} \\
\hline Small orders & 2.03 & $2.21^{* *}$ & 1.98 & $2.12^{\star *}$ \\
\hline Medium orders & 2.16 & $2.38^{* *}$ & 2.08 & $2.22^{* *}$ \\
\hline Large orders & 2.49 & 2.70 & 2.31 & $2.47^{* *}$ \\
\hline All & 2.05 & $2.29^{* *}$ & 2.07 & $2.25^{\star *}$ \\
\hline
\end{tabular}

Data are taken from the NYSE's System Order Database file over 6/30/97-7/11/97 and include orders from stocks identified in the CRSP names structure as ordinary common shares. Orders entered when the NBBO was unavailable (e.g., the open) or was less than $\$ 1 / 16$ or greater than $\$ 1 / 4$ were excluded. Small orders are for 100-500 shares. Medium orders are for 501-5,000 shares. Large orders are for more than 5,000 shares. Order Size $\leq$ Depth refers to orders with size less than or equal to the posted depth. Order Size $>$ Depth refers to orders with size greater than the posted depth. The short-run volatility is defined as $10,000 \times$ squared log return from the opening trade to the order submission time NBBO midpoint. The long-run volatility is the standard deviation of returns over the previous year for each stock. * and ** indicate the difference in volatility across order types is significant at the $5 \%$ and $1 \%$ levels, respectively, using a $t$-test.

\section{Are Investors Pursuing Optimal Order Submission Strategies?}

Under the null hypothesis of no order submission strategy, the selection of order type is assumed to be random. To determine if investors are pursuing optimal order submission strategies then one need only compare the average trading costs for each strategy. However, Tables 1-4 indicate order submission strategy may not be random. This becomes an issue in assessing whether investors are pursuing optimal order submission strategies for at least two reasons. First, the order type decision may be influenced by stock or market characteristics. Comparing the performance of each strategy under different circumstances may result in an unfair test of the performance of the two order types. Second, we would like to use a model to evaluate trading costs under each strategy. After fitting the model, the parameter estimates could be used to determine which order strategy is better, holding all else equal. However, if a selection bias exists, the parameter estimates of the model may be inconsistent. Using the inconsistent model, parameter es- 
timates may result in false inferences on the estimates of trading costs using the alternate strategy.

We use the method of matching and the Heckman two-step estimation to control for potential selection biases to ensure robustness of our results. The basic procedure is as follows. First, two subsamples are randomly formed. One subsample is used for parameter estimation and the other subsample is used for evaluation. A binomial probit modeling the choice of using the marketable limit order strategy is estimated. Next, the output from the probit is used to control for selectivity in estimating conditional trading costs as described below.

\section{A. Predicting Order Type Using the Probit Regression} ders as ${ }^{4}$

Relative effective spreads are modeled for market and marketable limit or-

$$
\begin{aligned}
\operatorname{RELSPREAD}_{i}^{\text {Limit }} & =\beta^{\text {Limit }}{ }^{\prime} X_{i}+\varepsilon_{i}^{\text {Limit }}, \\
\operatorname{RELSPREAD}_{i}^{\text {Market }} & =\beta^{\text {Market }}{ }^{\prime} X_{i}+\varepsilon_{i}^{\text {Market }},
\end{aligned}
$$

where $X_{i}$ is a vector of conditioning variables for each order $i, \beta$ is a vector of parameters to be estimated, and the $\varepsilon$ s are error terms. We assume the difference in expected execution costs across order types is a factor that determines the order type selected by a trader. The difference in expected relative effective spreads is

$$
\begin{aligned}
y_{i}^{*} & =E\left\{\operatorname{RELSPREAD}_{i}^{\text {Limit }}-\operatorname{RELSPREAD}_{i}^{\text {Market }} \mid X_{i}\right\} \\
& =\left(\beta^{\text {Limit }^{\prime}}-\beta^{\text {Market }^{\prime}}\right) X_{i}+\varepsilon_{i}^{\text {Limit }}-\varepsilon_{i}^{\text {Market }} \\
& =\gamma^{\prime} X_{i}+\zeta_{i} .
\end{aligned}
$$

In this model, trader $i$ chooses to submit a marketable limit order if $y_{i}^{*} \leq 0$, i.e., a marketable limit order strategy is expected to be less costly. The order type decision rule for an investor wishing to trade is

$$
\begin{aligned}
& y_{i}=1 \text { if } y_{i}^{*} \leq 0, \\
& y_{i}=0 \text { if } y_{i}^{*}>0,
\end{aligned}
$$

with $y_{i}=1$ for a marketable limit order and $y_{i}=0$ for a market order.

We use several independent variables to model the choice of order type in a probit framework. Because the bid-ask spread may be an important determinant of order submission strategy, we include three binary variables to control for the size of bid-ask spread at order arrival (with the base case being defined as having the spread equal to $\$ 1 / 4)$. SprdEq $\$ 1 / 16$ is a binary variable set to one if the bidask spread is $\$ 1 / 16$ at order arrival, otherwise it is zero. SprdEq $\$ 1 / 8$ is a binary variable set to one if the bid-ask spread is $\$ 1 / 8$ at order arrival, otherwise it is zero. SprdEq $\$ 3 / 16$ is a binary variable set to one if the bid-ask spread is $\$ 3 / 16$ at order arrival, otherwise it is zero. LogRelOrdSiz is the log of the order size divided by the opposite side quoted depth. SRVolatility and LRVolatility are the

\footnotetext{
${ }^{4}$ The effective spread was also considered and results were similar.
} 
Short-Run Volatility and the Long-Run Volatility, respectively, as defined above and in Table 4. Imbalance is the quote imbalance as described above and in Table 3. To pool buy and sell orders in a single regression, Imbalance is multiplied by -1 for sell orders. LogMktCap is the log of the market capitalization (in millions) of the underlying stock and is included to control for the liquidity of the stock. Buy is a dummy variable set to one if the order is a buy order, otherwise it is zero. Buy is included because buy orders may be more informative than sell orders for the reason that sell orders may include more liquidity motivated trades.

Table 5 reports the results from the probit regression, estimating the probability an investor chooses the marketable limit order strategy. That is,

$$
\operatorname{Pr}\left\{y_{i}=1\right\}=\Phi\left(\gamma^{\prime} X_{i}\right),
$$

where $\Phi$ is the cumulative distribution function of the standard normal. In general, the data support the univariate results. Likelihood ratio tests strongly reject the hypothesis the explanatory variables have zero coefficients.

For orders from non-individual investors (panel A) and individual investors (panel B), the probit results indicate limit orders are used more when the spread at order submission is smaller. ${ }^{5}$ This result is consistent with Harris (1997). Limit orders appear to be used more often as the order size increases relative to the depth as the coefficient estimates on LogRelOrdSiz are all positive and significant. Depending on the investor type, LRVolatility is either positively related to or negatively related to the use of limit orders. These observations and the coefficient estimates on SRVolatility are consistent with Table 4. Buy orders tend to be limit orders more often than sell orders, which may result from the differential informativeness of buy orders vs. sell orders. Orders for more liquid stocks, as proxied by LogMktCap, tend to be market orders proportionally more often.

The next step is to multiply the parameter estimates from the probit, $\gamma$, with the complementary set of observations, $X$, (i.e., the evaluation subsample) to estimate the probability of choosing a limit order $\left(\Phi\left(\gamma^{\prime} X\right)\right)$.

\section{B. Evaluating Conditional Execution Costs Using the Method of Matching}

The first method used to control for selectivity bias is the method of matching. The method of matching allows for a reduction in the selection bias as matches are constructed on the basis of observed characteristics, $X$. Rosenbaum and Rubin (1983) have shown that if matching on $X$ is valid, so is matching solely on the probability of selection. Thus, a multi-dimensional matching problem can be recast as a one-dimensional problem. This allows us to control for certain stock and market characteristics that may lead to a selection bias.

In this approach, we average the relative effective spreads for each order type over an interval of $\Phi\left(\gamma^{\prime} X_{i}\right)$ values. For more details on this procedure see Heckman, Ichimura, and Todd (1997). Here, the predicted probabilities of selecting the limit order strategy, $P_{i}\left(=\Phi\left(\gamma^{\prime} X_{i}\right)\right)$, are ranked, forming 20 portfolios. Differences in transactions costs for each portfolio are tested for significance using a $t$-test.

\footnotetext{
${ }^{5}$ In fact, $62 \%, 27 \%, 6 \%$, and $4 \%$ of all marketable limit orders were placed when the spread was $\$ 1 / 16, \$ 1 / 8, \$ 3 / 16$, and $\$ 1 / 4$, respectively.
} 
TABLE 5

Probit (1st Stage) Regressions of Selecting a Marketable Limit Order

\begin{tabular}{|c|c|c|c|c|}
\hline \multirow[b]{2}{*}{ Coefficient } & \multicolumn{2}{|c|}{ Order Size $\leq$ Depth } & \multicolumn{2}{|c|}{ Order Size > Depth } \\
\hline & $\begin{array}{l}\text { Parameter } \\
\text { Estimate } \\
\end{array}$ & $\begin{array}{c}\text { Standard } \\
\text { Error } \\
\end{array}$ & $\begin{array}{l}\text { Parameter } \\
\text { Estimate } \\
\end{array}$ & $\begin{array}{c}\text { Standard } \\
\text { Error }\end{array}$ \\
\hline \multicolumn{5}{|c|}{ Panel A. Orders from Non-Individual Investors } \\
\hline Intercept & $-0.204^{\star \star}$ & 0.012 & $-0.646^{\star \star}$ & 0.023 \\
\hline SprdEq $\$ 1 / 16$ & $0.826^{\star \star}$ & 0.009 & $0.750^{\star \star}$ & 0.016 \\
\hline SprdEq $\$ 1 / 8$ & $0.453^{\star *}$ & 0.009 & $0.405^{\star *}$ & 0.017 \\
\hline SprdEq $\$ 3 / 16$ & $0.087^{\star *}$ & 0.011 & 0.001 & 0.020 \\
\hline LogRelOrdSiz & $0.383^{* *}$ & 0.002 & $0.104^{\star \star}$ & 0.005 \\
\hline SRVolatility & -0.000 & 0.000 & $-0.001^{\star *}$ & 0.000 \\
\hline LRVolatility & $-7.963^{\star \star}$ & 0.264 & $-3.977^{\star *}$ & 0.542 \\
\hline Imbalance & $0.021^{* *}$ & 0.002 & 0.009 & 0.005 \\
\hline LogMktCap & $-0.034^{* *}$ & 0.001 & $-0.063^{\star *}$ & 0.003 \\
\hline Buy & $0.078^{* *}$ & 0.004 & $0.115^{\star \star}$ & 0.008 \\
\hline Pseudo $R^{2}$ & 0.174 & & 0.053 & \\
\hline \multicolumn{5}{|c|}{ Panel B. Orders from Individual Investors } \\
\hline Intercept & $-2.029^{\star *}$ & 0.024 & $-1.648^{* *}$ & 0.061 \\
\hline SprdEq $\$ 1 / 16$ & $0.525^{\star \star}$ & 0.017 & $0.472^{\star \star}$ & 0.040 \\
\hline SprdEq $\$ 1 / 8$ & $0.313^{* *}$ & 0.017 & $0.319^{* *}$ & 0.041 \\
\hline SprdEq $\$ 3 / 16$ & 0.005 & 0.022 & -0.010 & 0.051 \\
\hline LogRelOrdSiz & $0.137^{\star *}$ & 0.003 & $0.295^{\star *}$ & 0.016 \\
\hline SRVolatility & $0.002^{* *}$ & 0.000 & 0.000 & 0.000 \\
\hline LRVolatility & $6.919^{\star *}$ & 0.389 & $4.217^{\star *}$ & 1.075 \\
\hline Imbalance & $-0.058^{\star *}$ & 0.004 & $-0.176^{\star \star}$ & 0.013 \\
\hline LogMktCap & $-0.102^{\star \star}$ & 0.002 & $-0.092^{\star \star}$ & 0.007 \\
\hline Buy & $0.040^{* *}$ & 0.008 & $0.076^{\star *}$ & 0.023 \\
\hline Pseudo $R^{2}$ & 0.040 & & 0.064 & \\
\hline
\end{tabular}

The sample is described in Table 1. Parameter estimates are obtained using a random subsample of approximately half of all observations. SprdEq $\$ 1 / 16$ is equal to 1 when the spread at order submission is $\$ 1 / 16$, otherwise 0 . SprdEq $\$ 1 / 8$ is equal to 1 when the spread at order submission is $\$ 1 / 8$, otherwise 0 . SprdEq $\$ 3 / 16$ is equal to 1 when the spread at order submission is equal to $\$ 3 / 16$, otherwise 0 . LogRelOrdSiz is the log of the order size divided by the depth. SRVolatility is defined as $10,000 \times$ squared log return from the opening trade to the order submission time NBBO midpoint. LRVolatility is the standard deviation of returns in the previous year. Imbalance is described in Table 3. For sell orders, Imbalance is multiplied by -1 . LogMktCap is the log of the market capitalization in millions of dollars. Buy is a dummy variable set to 1 for buy orders, 0 otherwise. Order Size $\leq$ Depth refers to orders with size less than or equal to the opposite side posted depth. Order Size $>$ Depth refers to orders with size greater than the opposite side posted depth. * and ${ }^{* *}$ indicate significance at the $5 \%$ and $1 \%$ levels, respectively. Values indicated as 0.000 or -0.000 have been rounded to thousandths.

Table 6 reports the average relative effective spread by order type for each probability portfolio. Panel A reports the results for the orders from non-individual investors. First, some observations regarding the orders for fewer shares than are displayed at the opposite side quote. For the orders least likely to be limit orders, portfolio 1, the average relative effective spread is $0.138 \%$ and $0.259 \%$ for market and marketable limit orders, respectively. The difference of $0.121 \%$ is statistically significant at the $1 \%$ level. To put this number in dollar terms, given the average stock price on the NYSE in 1997 was $\$ 43.30$ (source: NYSE Fact Book), the $0.121 \%$ difference represents an additional cost of about $5 \notin$ on a round-trip. Therefore the difference in trading costs is economically significant as well.

At the other end of the spectrum, the portfolio of orders most likely to be limit orders, portfolio 20, indicates market orders are more expensive $(0.221 \%$ vs. $0.181 \%$ ). The difference in relative effective spreads generally increases from portfolio 1 to portfolio 20 . These data are consistent with the average market order 
TABLE 6

Relative Effective Spreads (\%) for Portfolios Ranked on the Estimated Likelihood of Using a Limit Order Strategy

\begin{tabular}{|c|c|c|c|c|c|c|}
\hline \multirow[b]{2}{*}{ Portfolio } & \multicolumn{3}{|c|}{ Order Size $\leq$ Depth } & \multicolumn{3}{|c|}{ Order Size > Depth } \\
\hline & Market & Limit & Difference & Market & Limit & Difference \\
\hline \multicolumn{7}{|c|}{ Panel A. Orders from Non-Individual Investors } \\
\hline 1 (Least likely) & $0.138 \%$ & $0.259 \%$ & $-0.121 \%$ ** & $0.241 \%$ & $0.354 \%$ & $-0.083 \%$ ** \\
\hline 2 & 0.161 & 0.256 & $-0.095^{\star \star}$ & 0.434 & 0.467 & $-0.033^{* \star}$ \\
\hline 3 & 0.164 & 0.245 & $-0.081^{* *}$ & 0.560 & 0.550 & 0.010 \\
\hline 4 & 0.175 & 0.226 & $-0.051^{* *}$ & 0.455 & 0.445 & 0.010 \\
\hline 5 & 0.186 & 0.237 & $-0.051^{* *}$ & 0.348 & 0.341 & 0.007 \\
\hline 6 & 0.193 & 0.238 & $-0.045^{\star *}$ & 0.353 & 0.351 & 0.002 \\
\hline 7 & 0.199 & 0.219 & $-0.020^{\star *}$ & 0.366 & 0.369 & -0.003 \\
\hline 8 & 0.210 & 0.201 & 0.009 & 0.383 & 0.348 & $0.035^{\star *}$ \\
\hline 9 & 0.211 & 0.206 & 0.005 & 0.378 & 0.327 & $0.051^{\star *}$ \\
\hline 10 & 0.221 & 0.219 & 0.002 & 0.368 & 0.308 & $0.060^{\star *}$ \\
\hline 11 & 0.224 & 0.219 & 0.005 & 0.320 & 0.280 & $0.040^{* *}$ \\
\hline 12 & 0.220 & 0.218 & 0.002 & 0.288 & 0.257 & $0.031^{\star *}$ \\
\hline 13 & 0.210 & 0.188 & $0.022^{\star *}$ & 0.302 & 0.275 & $0.027^{*}$ \\
\hline 14 & 0.213 & 0.162 & $0.051^{\star *}$ & 0.313 & 0.280 & $0.033^{*}$ \\
\hline 15 & 0.210 & 0.188 & $0.022^{* *}$ & 0.324 & 0.284 & $0.040^{* *}$ \\
\hline 16 & 0.219 & 0.190 & $0.029^{* *}$ & 0.351 & 0.309 & $0.042^{* *}$ \\
\hline 17 & 0.222 & 0.205 & $0.017^{* *}$ & 0.344 & 0.338 & 0.006 \\
\hline 18 & 0.212 & 0.175 & $0.037^{\star *}$ & 0.447 & 0.351 & $0.096^{\star \star}$ \\
\hline 19 & 0.202 & 0.159 & $0.043^{\star *}$ & 0.522 & 0.457 & $0.065^{\star *}$ \\
\hline 20 (Most likely) & 0.221 & 0.181 & $0.040^{* *}$ & 0.735 & 0.639 & $0.096^{* *}$ \\
\hline \multicolumn{7}{|c|}{ Panel B. Orders from Individual Investors } \\
\hline 1 (Least likely) & $0.092 \%$ & $0.068 \%$ & $0.004 \%$ & $0.285 \%$ & $0.260 \%$ & $0.025 \%$ \\
\hline 2 & 0.095 & 0.093 & 0.002 & 0.381 & 0.330 & 0.051 \\
\hline 3 & 0.110 & 0.122 & -0.012 & 0.387 & 0.293 & $0.094^{*}$ \\
\hline 4 & 0.123 & 0.136 & -0.013 & 0.433 & 0.462 & -0.021 \\
\hline 5 & 0.135 & 0.116 & 0.019 & 0.486 & 0.475 & 0.011 \\
\hline 6 & 0.157 & 0.179 & -0.022 & 0.463 & 0.408 & 0.055 \\
\hline 7 & 0.169 & 0.201 & $-0.032^{\star *}$ & 0.475 & 0.412 & 0.063 \\
\hline 8 & 0.186 & 0.210 & -0.024 & 0.546 & 0.457 & 0.089 \\
\hline 9 & 0.208 & 0.262 & $-0.054^{\star *}$ & 0.529 & 0.456 & 0.073 \\
\hline 10 & 0.223 & 0.272 & $-0.049^{\star \star}$ & 0.514 & 0.480 & 0.034 \\
\hline 11 & 0.238 & 0.287 & $-0.049^{* *}$ & 0.528 & 0.547 & -0.019 \\
\hline 12 & 0.253 & 0.290 & $-0.037^{\star *}$ & 0.616 & 0.538 & 0.078 \\
\hline 13 & 0.274 & 0.327 & $-0.053^{\star *}$ & 0.621 & 0.599 & 0.022 \\
\hline 14 & 0.286 & 0.334 & $-0.048^{\star *}$ & 0.655 & 0.627 & 0.028 \\
\hline 15 & 0.307 & 0.347 & $-0.040^{\star *}$ & 0.738 & 0.667 & 0.071 \\
\hline 16 & 0.330 & 0.387 & $-0.057^{\star *}$ & 0.648 & 0.699 & -0.051 \\
\hline 17 & 0.351 & 0.435 & $-0.084^{\star *}$ & 0.821 & 0.710 & 0.111 \\
\hline 18 & 0.400 & 0.440 & $-0.040^{*}$ & 0.840 & 0.813 & 0.027 \\
\hline 19 & 0.477 & 0.533 & $-0.056^{\star *}$ & 1.053 & 0.843 & 0.210 \\
\hline 20 (Most likely) & 1.006 & 1.178 & $-0.172^{\star *}$ & 1.637 & 1.547 & 0.090 \\
\hline
\end{tabular}

The spread is based on the NBBO at order arrival time. Data are taken from 6/30/97-7/11/97 and include only those shares in the CRSP names structure that are ordinary common shares. Parameter estimates are obtained using a random subsample of approximately half of all observations. The complementary subsample is used to form portfolios and calculate average relative effective spreads (in percent) used in this table. Order Size $\leq$ Depth refers to orders with size less than or equal to the posted depth. Order Size $>$ Depth refers to orders with size greater than the posted depth. ${ }^{*}$ and ${ }^{* *}$ indicate the difference is significant at the $5 \%$ and $1 \%$ levels, respectively, using a $t$-test.

being less expensive when market orders are the primary order type, and with the average market order being more expensive when marketable limit orders are the primary order type.

Consider next the orders for more shares than are at the posted depth. As above, the data indicate the difference in trading costs increases with the likelihood of limit order usage. With the exception of the two portfolios most likely to 
be market orders, these data are consistent with the marketable limit order strategy, on average, being superior when the order size exceeds the quoted depth.

Panel B of Table 6 reports the results for orders from individual investors. Surprisingly, it appears marketable limit orders are, in general, more expensive than market orders when the order size is less than or equal to the opposite side quoted depth. Notably, costs diverge as the likelihood of a marketable limit order submission increases. These results are inconsistent with the average individual investor benefiting (in terms of lower trading costs) from using the marketable limit order strategy. Alternatively, it may be that our probit model is mis-specified due to omitted variables. For example, commissions may vary across order types for individual investors, but we cannot observe this variable. When the order size is greater than the depth, the data do not generally reveal significant cost differences across order types.

\section{Estimating Conditional Execution Costs Using the Heckman Method}

The Heckman (1979) method is commonly used to handle selection bias problems. In the second stage of this method, the probit probability estimates are used to control for the selection bias. We wish to use this method for two reasons. First, we would like to be able to statistically confirm the existence of the apparent selection bias. The Heckman method can detect such a bias in a rather straightforward fashion. Thus, this method allows for a robustness check. Second, we would like to use the model to estimate trading costs under the current and alternate strategies. The Heckman method provides consistent parameters in our cost model described below. These parameters can be used to examine the tradeoffs in order submission strategies, something that cannot be done without the correction for selectivity.

Because limit orders are observed only when $y_{i}^{*} \leq 0$, the error term $\varepsilon_{i}^{\text {Limit }}$ does not have a zero mean, conditional on being a limit order. The conditional expected trading costs for marketable limit orders and market orders are denoted as

$$
\begin{aligned}
E\left\{\operatorname{RELSPREAD}_{i}^{\text {Limit }} \mid y^{*} \leq 0\right\}= & \beta^{\text {Limit }^{\prime}} X_{i}+\lambda_{\text {Limit }}\left[\phi\left(\gamma^{\prime} X_{i}\right) / \Phi\left(\gamma^{\prime} X_{i}\right)\right] \\
E\left\{\operatorname{RELSPREAD}_{i}^{\text {Market }} \mid y^{*}>0\right\}= & \beta^{\text {Market }^{\prime}} X_{i} \\
& +\lambda_{\text {Market }}\left[\phi\left(\gamma^{\prime} X_{i}\right) /\left(\Phi\left(\gamma^{\prime} X_{i}\right)-1\right)\right]
\end{aligned}
$$

where $\phi\left(\gamma^{\prime} X_{i}\right)$ and $\Phi\left(\gamma^{\prime} X_{i}\right)$ are, respectively, the density and cumulative distribution function of the standard normal evaluated at $\gamma^{\prime} X_{i}$, and $\lambda$ is the covariance between $\varepsilon_{i}$ and $\zeta_{i}$. In this methodology, estimating the second stage equation by OLS provides consistent estimates of the parameters. The estimated coefficient of the selectivity variable, $\lambda$, provides useful information about the extent to which the order type selection and execution costs are interrelated. This regression provides an opportunity to test the null hypothesis that there is no order submission strategy, i.e., $\lambda=0$. Greene (1981) notes there is a problem with this estimation procedure as the standard errors in the second stage may be incorrect since the selectivity variables are themselves estimates. Greene (1981) outlines methods to correct this problem. 
Table 7 provides the second-step OLS estimates of the parameters with the relative effective spread as the dependent variable. Panel A presents results for orders from non-individual investors. When the order size is less than or equal to the opposite side depth, the coefficient estimate on the selectivity variable, Lambda, is insignificant for marketable limit orders and positive for market orders. We interpret this result in the following way. Marketable limit order traders, holding all else equal, would not incur higher costs by switching order types. However, arbitrarily choosing the market order strategy, without regard to the underlying stock or market conditions, would, on average, result in a higher relative effective spread as the likelihood of a marketable limit order submission increases.

TABLE 7

OLS (Second Stage) Regressions Controlling for Selection Bias

Panel A. Orders from Non-Individual Investors

\begin{tabular}{|c|c|c|c|c|c|c|c|c|}
\hline \multirow[b]{3}{*}{ Parameter } & \multicolumn{4}{|c|}{ Order Size $\leq$ Depth } & \multicolumn{4}{|c|}{ Order Size > Depth } \\
\hline & \multicolumn{2}{|c|}{ Marketable Limit Order } & \multicolumn{2}{|c|}{ Market Order } & \multicolumn{2}{|c|}{ Marketable Limit Order } & \multicolumn{2}{|c|}{ Market Order } \\
\hline & $\begin{array}{l}\text { Parameter } \\
\text { Estimate } \\
(\times 1000)\end{array}$ & $\begin{array}{l}\text { Standard } \\
\text { Error } \\
(\times 1000)\end{array}$ & $\begin{array}{l}\text { Parameter } \\
\text { Estimate } \\
(\times 1000)\end{array}$ & $\begin{array}{c}\text { Standard } \\
\text { Error } \\
(\times 1000)\end{array}$ & $\begin{array}{c}\text { Parameter } \\
\text { Estimate } \\
(\times 1000)\end{array}$ & $\begin{array}{l}\text { Standard } \\
\text { Error } \\
(\times 1000)\end{array}$ & $\begin{array}{c}\text { Parameter } \\
\text { Estimate } \\
(\times 1000)\end{array}$ & $\begin{array}{c}\text { Standard } \\
\text { Error } \\
(\times 1000)\end{array}$ \\
\hline Intercept & $-2.40^{* *}$ & 0.25 & $-1.60^{* *}$ & 0.11 & $1.45^{\star \star}$ & 2.33 & $3.40^{\text {** }}$ & 1.03 \\
\hline SprdEq $\$ 1 / 16$ & $-1.71^{* *}$ & 0.14 & -0.12 & 0.07 & $-9.54^{\star *}$ & 0.89 & $7.52^{\star *}$ & 1.37 \\
\hline SprdEq $\$ 1 / 8$ & $-1.14^{* *}$ & 0.09 & $-0.33^{\star *}$ & 0.04 & $-5.68^{* *}$ & 0.51 & $3.66^{* *}$ & 0.71 \\
\hline SprdEq\$3/16 & $-0.67^{* \star}$ & 0.05 & $-0.60^{*}$ & 0.04 & $-0.86^{\star *}$ & 0.11 & $-0.96^{\star *}$ & 0.10 \\
\hline LogRelOrdSiz & -0.10 & 0.06 & $0.32^{\star *}$ & 0.03 & -0.20 & 0.11 & $2.16^{\star *}$ & 0.20 \\
\hline SRVolatility & $0.04^{\star \star}$ & 0.00 & $-0.07^{\star \star}$ & 0.00 & $0.06^{\star \star}$ & 0.00 & $0.02^{\star *}$ & 0.00 \\
\hline LRVolatility & $80.67^{* *}$ & 1.61 & $81.68^{* *}$ & 1.20 & $151.38^{* *}$ & 4.85 & $20.68^{\star \star}$ & 8.10 \\
\hline Imbalance & $0.21^{\star *}$ & 0.01 & $0.44^{\star *}$ & 0.01 & $0.08^{\star *}$ & 0.02 & $0.38^{\star *}$ & 0.03 \\
\hline LogMktCap & $-0.82^{* *}$ & 0.01 & $-0.80^{* *}$ & 0.01 & $-0.64^{\star *}$ & 0.07 & $-2.03^{* *}$ & 0.12 \\
\hline Buy & 0.01 & 0.02 & $0.18^{* *}$ & 0.02 & $-0.96^{\star \star}$ & 0.13 & $1.52^{* *}$ & 0.22 \\
\hline Lambda & -0.06 & 0.26 & $1.51^{\star *}$ & 0.14 & $-15.51^{\star *}$ & 1.99 & $18.70^{* *}$ & 2.75 \\
\hline $\begin{array}{l}\text { Adjusted } R^{2} \\
F \text {-Value }\end{array}$ & $\begin{array}{c}0.35 \\
10,490^{\star *}\end{array}$ & & $\begin{array}{c}0.18 \\
5,512^{\star \star}\end{array}$ & & $\begin{array}{c}0.30 \\
2,490^{\star *}\end{array}$ & & $\begin{array}{c}0.26 \\
1,272^{\star *}\end{array}$ & \\
\hline \multicolumn{9}{|c|}{ Panel B. Orders from Individual Investors } \\
\hline Parameter & $\begin{array}{c}\text { Parameter } \\
\text { Estimate } \\
(\times 100) \\
\end{array}$ & $\begin{array}{l}\text { Standard } \\
\text { Error } \\
(\times 100)\end{array}$ & $\begin{array}{c}\text { Parameter } \\
\text { Estimate } \\
(\times 100) \\
\end{array}$ & $\begin{array}{c}\text { Standard } \\
\text { Error } \\
(\times 100) \\
\end{array}$ & $\begin{array}{c}\text { Parameter } \\
\text { Estimate } \\
(\times 100) \\
\end{array}$ & $\begin{array}{l}\text { Standard } \\
\text { Error } \\
(\times 100)\end{array}$ & $\begin{array}{c}\text { Parameter } \\
\text { Estimate } \\
(\times 100) \\
\end{array}$ & $\begin{array}{c}\text { Standard } \\
\text { Error } \\
(\times 100) \\
\end{array}$ \\
\hline Intercept & $-52.05^{\star \star}$ & 1.32 & $-0.41^{\star *}$ & 0.01 & $-22.69^{\star *}$ & 2.11 & $-0.66^{\star \star}$ & 0.05 \\
\hline SprdEq \$1/16 & $9.00^{\star *}$ & 0.24 & $-0.68^{\star *}$ & 0.01 & $3.27^{\star \star}$ & 0.37 & $-1.95^{\star \star}$ & 0.10 \\
\hline SprdEq $\$ 1 / 8$ & $5.29^{* *}$ & 0.15 & $-0.43^{\star *}$ & 0.01 & $2.14^{* *}$ & 0.26 & $-1.33^{\star *}$ & 0.07 \\
\hline SprdEq $\$ 3 / 16$ & $-0.14^{\star \star}$ & 0.04 & $-0.10^{\star *}$ & 0.01 & $-0.24^{\star *}$ & 0.09 & $-0.24^{\star \star}$ & 0.03 \\
\hline LogRelOrdSiz & $2.37^{* *}$ & 0.06 & $-0.18^{\star *}$ & 0.00 & $2.32^{\star \star}$ & 0.22 & $-0.85^{\star \star}$ & 0.07 \\
\hline SRVolatility & $0.03^{\text {** }}$ & 0.00 & $-0.00^{\star *}$ & 0.00 & 0.00 & 0.00 & 0.00 & 0.00 \\
\hline LRVolatility & $139.75^{* *}$ & 3.07 & $-1.20^{\star *}$ & 0.20 & $69.27^{\star *}$ & 3.45 & $4.81^{\text {** }}$ & 1.29 \\
\hline Imbalance & $-0.91^{\star \star}$ & 0.03 & $0.14^{\star \star}$ & 0.00 & $-1.31^{\star \star}$ & 0.13 & $0.65^{\star \star}$ & 0.04 \\
\hline LogMktCap & $-1.96^{* *}$ & 0.05 & $0.02^{* *}$ & 0.00 & $-0.93^{\star *}$ & 0.07 & $0.12^{\star *}$ & 0.02 \\
\hline Buy & $0.81^{\star \star}$ & 0.02 & $-0.04^{\star *}$ & 0.00 & $0.65^{\star \star}$ & 0.07 & $-0.24^{\star \star}$ & 0.02 \\
\hline Lambda & $21.58^{\star * *}$ & 0.56 & $-4.24^{\star *}$ & 0.06 & $10.79^{\star *}$ & 1.08 & $-7.02^{\star *}$ & 0.42 \\
\hline Adjusted $R^{2}$ & 0.30 & & 0.21 & & 0.37 & & 0.28 & \\
\hline$F$-Value & $924^{* *}$ & & $4,324^{\star *}$ & & $266^{\star \star}$ & & $374^{* *}$ & \\
\hline
\end{tabular}

The sample is described in Table 1. Parameter estimates are obtained using a random subsample of approximately half of all observations. SprdEq $\$ 1 / 16$ is equal to 1 when the spread at order submission is $\$ 1 / 16$, otherwise 0 . SprdEq $\$ 1 / 8$ is equal to 1 when the spread at order submission is $\$ 1 / 8$, otherwise 0 . SprdEq $\$ 3 / 16$ is equal to 1 when the spread at order submission is equal to $\$ 3 / 16$, otherwise 0 . LogRelOrdSiz is the log of the order size divided by the depth. SRVolatility is the range of stock prices for the current day. LRVolatility is the standard deviation of returns in the previous year. Imbalance is described in Table 3. For sell orders, Imbalance is multiplied by -1 . LogMktCap is the log of the market capitalization in millions of dollars. Buy is a dummy variable set to 1 for buy orders, 0 otherwise. Order Size $\leq$ Depth refers to orders with size less than or equal to the posted depth. Order Size $>$ Depth refers to orders with size greater than the posted depth. ${ }^{*}$ and ${ }^{* *}$ indicate significance at the $5 \%$ and $1 \%$ levels, respectively. Values indicated as 0.00 or -0.00 have been rounded to hundredths. 
For the marketable limit order regression when the order size is for more shares than are at the opposite side quoted depth, Lambda is significantly less than zero, indicating relative costs decrease with increases in the likelihood of choosing a marketable limit order. The opposite is true for the market order regression. That is, relative costs of market orders increase with increases in the likelihood of choosing a marketable limit order.

Table 7, panel B, provides the results for the orders from individual investors. The coefficient estimates on Lambda are all significant, indicating a selection bias is present. However, the signs are generally opposite of the same variable in panel A. These results are generally consistent with those in Table 6, panel B, and show the marketable limit order strategy is more expensive, holding the selected variables constant.

\section{The Impact of Switching Strategies}

The parameters from the second stage regressions are next used to estimate the trading costs of the observations in the evaluation subsample for the selected order submission strategy and the alternate order submission strategy. Each observation in the evaluation subsample is multiplied by the appropriate market and marketable limit parameter vectors reported in Table 7. The products provide estimated relative effective spreads for the selected strategy and the alternate strategy.

Table 8 reports the average estimated costs of the selected and alternate strategies, as well as the impact of switching order types. Regardless of investor type, the average trader chooses the less costly strategy when the order size is less than the depth. For example, non-individual investors submitting orders for fewer shares than are at the opposite side quoted depth, pay an average estimated relative effective spread of $0.196 \%$. Using the alternate strategy, the average estimated relative effective spread is $0.212 \%$. The difference of $0.016 \%$ is statistically significant. One could debate whether that amount is economically significant. For a 1,000 share order of a stock priced at $\$ 50$, the difference would amount to $\$ 8$. This difference may be smaller than the difference in commissions for submitting a market or a limit order. However, we should point out the differences reported are averages. In some cases, the marginal costs of choosing the alternate order type could be substantially greater. When the order size exceeds the opposite side quoted depth, the average non-individual trader chooses the less costly order type. In the same situation, the average individual investor chooses the more costly order type. However, the difference of $0.006 \%$ is quite small economically.

This methodology also allows us to examine specific cases in which a suboptimal order submission strategy was used. For example, Table 8 shows trades from non-individual investors self-selected to use the market order strategy when the order size was greater than the opposite side quoted depth paid, on average, $0.023 \%$ higher transactions costs. This difference is statistically significant at the $1 \%$ level. To further investigate the orders that presumably used sub-optimal strategies, we examined those orders in which our model predicted relative effective spreads to be at least $0.1 \%$ cheaper using the alternate strategy. We found approximately $9 \%$ of the orders met this condition. Of these orders, approximately $18 \%$ came from non-individual investor accounts and $82 \%$ came from individual 
TABLE 8

Average Impact on Relative Effective Spread (\%) of Switching Order Submission Strategy

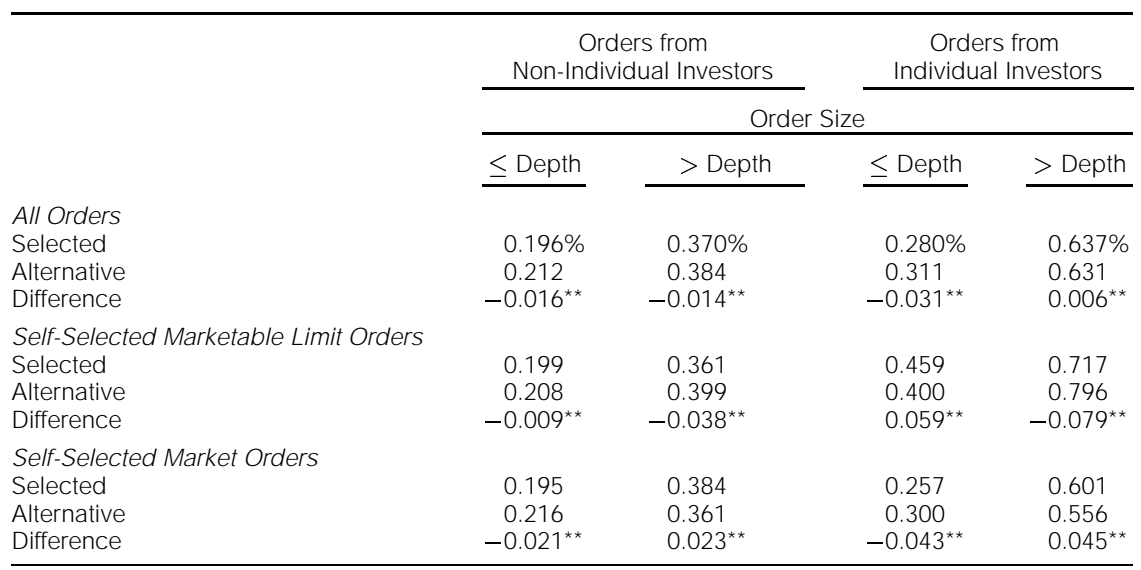

The sample is described in Table 1. Probit and OLS parameter estimates are obtained using a random subsample of approximately half of all observations. The complementary subsample is used to form portfolios (using the probit estimates) and calculate estimated relative effective spreads (using the OLS estimates) using the selected strategy and the alternate strategy. Order Size $\leq$ Depth refers to orders with size less than or equal to the posted depth. Order Size $>$ Depth refers to orders with size greater than the posted depth. * and ${ }^{* *}$ indicate significance at the $5 \%$ and $1 \%$ levels, respectively.

investor accounts. Because the number of orders from non-individual investors is 2.6 times the number of orders from individual investors (see Table 1), it may be better to report the likelihood of using the sub-optimal strategy. From this perspective, $2 \%(25 \%)$ of the orders from non-individual (individual) investors use a sub-optimal strategy. In summary, individual investors tend to use sub-optimal strategies more often than non-individuals.

\section{Conclusion}

This paper addresses a result that has persisted over time and is found on multiple stock exchanges. That is, unconditionally, marketable limit orders perform worse than market orders on a trading cost basis. Interestingly, the marketable limit order strategy continues to be used. We show investors self-select the order type based on current market conditions. Marketable limit orders are used more often for larger orders and instances where an order imbalance exists. After correcting for the selectivity bias, we find the average investor strategically selects the order type with lower expected trading costs. We provide evidence suggesting investors would not, on average, achieve better performance, and may achieve worse performance, if they arbitrarily switched order types. Additionally, we find data consistent with individual investors being less able to choose optimal strategies. However, this result may be due to an omitted variable effect. 


\section{References}

Angel, J. J. "Who Gets Price Improvement on the NYSE?” Working Paper, Georgetown Univ. (1997).

Bacidore, J. M.; R. H. Battalio; and R. H. Jennings. "Depth Improvement and Adjusted Price Improvement on the NYSE." Working Paper \#2000-04, NYSE (2000).

Biais, B.; P. Hillion; and C. Spatt. "An Empirical Analysis of the Limit Order Book and the Order Flow in the Paris Bourse." Journal of Finance, 50 (1995), 1655-1689.

Easley, D., and M. O'Hara. "Price, Trade Size, and Information in Securities Markets." Journal of Financial Economics, 19 (1987), 69-90.

Greene, W. "Sample Selection Bias as a Specification Error: Comment." Econometrica, 49 (1981), 795-798.

Handa, P.; R. A. Schwartz; and A. Tiwari. “The Economic Value of the Amex Trading Floor.” Working Paper, Univ. of Iowa (1999).

Harris, L. "Optimal Dynamic Order Submission Strategies in Some Stylized Trading Problems." Working Paper, Univ. of Southern California (1997).

Harris, L., and J. Hasbrouck. "Market vs. Limit Orders: The SuperDOT Evidence on Order Submission Strategy.” Journal of Financial and Quantitative Analysis, 31 (1996), 213-231.

Hasbrouck, J. "Using the TORQ Database." Working Paper, NYSE (1992).

Heckman, J. J. "Sample Selection Bias as a Specification Error.” Econometrica, 47 (1979), 153-162.

Heckman, J. J.; H. Ichimura; and P. Todd. "Matching as an Econometric Evaluation Estimator." Review of Economic Studies, 65 (1997), 261-294.

Holden, C. W., and S. Chakravarty. "An Integrated Model of Market and Limit Orders." Journal of Financial Intermediation, 4 (1995), 213-241.

Kumar, P., and D. J. Seppi. "Limit and Market Orders with Optimizing Traders." Working Paper, Carnegie Mellon (1992).

Madhavan, A., and M. Cheng. "In Search of Liquidity: Block Trades in the Upstairs and Downstairs Markets.” Review of Financial Studies, 10 (1997), 175-204.

Rosenbaum, P., and D. B. Rubin. "The Central Role of the Propensity Score in Observational Studies for Causal Effects.” Biometrika, 70 (1983), 41-55.

Ross, K. D.; J. E. Shapiro; and K. A. Smith. "Price Improvement of SuperDot Market Orders on the NYSE." Working Paper, NYSE (1996).

Sofianos, G., and I. Werner. "The Trades of NYSE Floor Brokers." Journal of Financial Markets, 3 (2000), 139-176.

U.S. SEC Report on the Practice of Preferencing Pursuant to Section 510(c) of the National Securities Markets Improvement Act of 1996 (April 11, 1997). 